\section{ENTEROCOLONIC CONDITIONS IN CHRONIC CONSTIPATION *}

\section{GRANVILLE S. HANES, M.D.} LOUISVILLE, KY.

The fact that constipation has been and is under continuous discussion shows conclusively the lack of perfect knowledge emanating from any source. In this paper I shall include stasis and obstipation under the general term of constipation.

No doubt there is an element of truth in the various explanations of constipation, but it may be safely asserted that many of these explanations require further elucidation. In dealing with this question the surgeon and the general practitioner are apparently arrayed against each other, but, as a matter of fact, the views of both contain much that is true. The great difficulty is for us to see every phase of this immense subject.

In the first place, some thought must be given the more elementary aspects of the question. The whole scheme of alimentation provides for the onward progress of the ingested food; at some points it is retarded, while at others it passes with considerable rapidity. Wherever the ingesta are normally retarded, we are most likely to find increased delay in the progress of the intestinal contents; therefore, it is at those points that we note the greatest tendency to constipation.

Since provision has been made for the food to pass easily through the lumen of the small intestine, we do not frequently find here conditions which give rise to constipation. The character of the food ingested, and the conditions controlling the intestinal, hepatic and pancreatic secretions, together with certain varieties of catarrh do, however, have some influence in the production of constipation. The hepatic secretions, catarrhal inflammations and foods must not be considered lightly in this connection.

The first portion of the intestinal tract where we observe stagnation of the contents is in the terminal ileum. At this point there exists an anatomic obstruction which materially retards the onward progress of the ingesta, the muscle fibers of the terminal ileum being thickened and thus offering greater resistance than the intestine above.

The next point offering obstruction is the ileocecal junction, the lumen here being diminished and also further constricted by circular muscular fibers which permit more perfect control of the ileocecal opening.

\section{CAUSATIVE FACTORS IN CONSTIPATION}

In the large intestine are found the chief causes of chronic constipation, the cecum and ascending colon being concerned either directly or indirectly in a large percentage of cases in which patients complain of this symptom. Many reasons may be adduced to explain the frequent stagnation in the right colon.

1. The anatomic arrangement of this segment of the colon is such that it naturally retards easy progress of its contents. The large intestine from the cecum to the sigmoid becomes progressively smaller, the variation between the two extremities being approximately three to one. Thus it may be seen that material forced from the cecum toward the sigmoid will require a pressure from behind many times greater than if the lumen were not diminished.

- Read before the Section on Gastro-Enterology and Proctology at the Sixty-Eighth Annual Session of the American Medical Association, New York, June, 1917.
2. The watery elements of the material emptied into the cecum are rapidly absorbed by the cecum and ascending colon. Because of the obstruction at the hepatic angle, the decrease in the colonic lumen, and the denser consistency of the feces, considerable force is required to propel the ingesta downward into the sigmoid.

3. A most potent reason for stasis in the cecum and ascending colon, and one which is generally overlooked or ignored, is the tightly contracted muscles controlling the terminal outlet of the intestinal tract. Not only are these powerful muscles in a state of tetanic contraction, but there is often sufficient irritation in the rectal and sigmoidal walls to cause an unnatural contraction, thus materially diminishing the normal capacity of these organs for the reception of feces. In every case of chronic constipation it is imperative that these organs be thoroughly investigated. At the time of operation for some rectal lesion, doubtless we have all noted both the sigmoid and rectum filled with water and feces, notwithstanding that a purgative had been administered twentyfour hours previously, and an enema not more than two or three hours before the operation. Again, we frequently hear patients say their "bowels are paralyzed," meaning that when they have a desire to defecate they cannot command sufficient force to expel the feces from the rectum and sigmoid. There is no paralysis in such cases, but on the contrary there is a spasticity which cannot be overcome by normal efforts.

4. There must be a normal balance of intracolonic pressure for defecation to occur normally. If there are contracted segments from irritation and the lumen is thus diminished, there must result a tendency to constipation. On the other hand, if the mucosa of the entire large intestine is actively inflamed, the walls become thickened and contracted, the lumen is materially diminished, an excessive amount of mucus is secreted, the feces are forced onward without time for absorption of the watery elements, and we then have the other extreme, namely, diarrhea.

5. Not infrequently the entire colon is found much larger than normal. Under such circumstances it is easy to understand that a given amount of material cannot pass through the colon with average rapidity when the lumen is large and the walls are unable to contract down on the contents. If the colonic lumen is comparatively small, which of necessity means thicker and more powerful muscle fibers, the contents will be propelled more certainly and effectively. The muscle fibers in the dilated colonic wall are attenuated and weak and, therefore, cannot contract on and move its contents forward with an equal degree of certainty.

6. It must also be conceded that a long transverse colon and redundant sigmoid present a physical condition more difficult for nature to cope with than when these parts of the bowel are of average normal length.

7. It seems useless to add that any growth in the intestinal lumen may cause obstruction and constipation, such as cancer, etc. Of course, as stated in the beginning of this paper, we include under the term constipation those conditions often referred to as stasis and obstipation.

With the feces in a liquid state a great deal of obstruction must be present to prevent fairly easy progress through the colon to the terminal outlet; it is the hard and formed fecal material which passes with difficulty along its course. We can readily under- 
stand how much more easily patients expel thin and unformed feces from the rectum than when the excreta is formed and hard; and the same holds true of the passage of fecal material along any portion of the intestinal tract.

I wish to remark at this juncture that the condition just mentioned is one of great importance. Many surgeons have performed colostomies in one way or another modifying to a greater or less degree certain points in the technic of the operation, and the patients had little difficulty in control through the artificial opening. The surgeon at once concluded that the modified technic in his operation was responsible for the excellent artificial control. Whatever that modification may have been, no technic has ever been devised whereby colostomy may be done and have any degree of satisfaction in artificial control, with the colonic contents in a liquid state. On the contrary, so far as control is concerned, almost any colostomy will be a success with the fecal contents well formed while passing through the transverse and descending colon.

The same deception applies to destruction of the sphincter muscles in operating for rectal cancer, etc. With the colonic contents dry and formed when passing downward through the sigmoid and into the rectum, any control at the terminal outlet by the sphincter muscles can scarcely be necessary. Owing to the deep hollow in the sacrum, and the provision made by the sigmoid for retaining the feces, exit is delayed until forced forward by the accessory muscles of defecation, aided by peristalsis.

Attention is called to the foregoing facts because they illustrate more forcibly than any other argument I could present, the importance of understanding the difference in the amount of power, required to move forward the colonic contents in liquid and solid forms.

8. In addition to the foregoing causative factors in constipation, it is my opinion that there is no element which plays a more important rôle than that of bacterial life. 'The organisms are so numerous, they thrive and multiply under so many varying circumstances, that it is impossible with our present understanding of germ life to properly estimate their full influence as causative factors either directly or indirectly in the production of constipation. The intestinal tract is so constructed that there is the least possible tendency for friction or the adherence of material to the mucosa. The greatest possible provision has been made for the easy movement of the ingesta by the polished epithelial surface of the mucosa, with the production of abundant mucus, which is unequaled as a lubricant.

The first effect that bacteria produce as a causative factor in constipation is an inflammatory reaction in the mucous membrane. The superficial cells become roughened by granulations, which has a marked effect in retarding the progress of the intestinal contents because of the disposition for material to adhere to the roughened surfaces. Many patients would be affected with pronounced constipation, when the mucous membrane is in this chronic state, were it not for the production of an abundance of mucus which compensates for this defect. When inflammation is active and mucus is produced in large quantities, and when the irritation is sufficient to cause contraction of the intestinal wall, we have the other extreme, namely, diarrhea. As will be mentioned later, a large percentage of constipated individuals give a history of having had, a number of years previously, an attack of some acute inflammatory disease involving the intestinal mucosa. These inflammatory areas are what might be considered the direct influences of bacteria on the intestinal mucosa.

The second influence of bacteria as factors in the production of constipation is on the musculature of the intestinal wall. As already suggested, the effect of very active bacterial life on the intestinal mucosa, whether there be ulceration or not, with the irritation of the nerve endings in the mucous membrane, the exaggerated contraction of the muscular wall, and an abundance of mucus, all taken together, produce diarrhea or dysentery. When there is gradual subsidence of the irritating effect of bacterial life on the tissues, then the intestinal walls begin to relax, the overstimulated mucous glands contract and produce a diminished amount of mucus, and the epithelial cells become granular from the chronic infection present. This stage, though months or years may have elapsed since the initiation of the infection, may be the beginning of chronic constipation. As the musculature relaxes under the influence of bacterial infection, with consequent increasing dilatation of the intestinal wall, an increased atrophy of the mucous glands, and also an increased granulation of the diseased epithelial cells, the constipation becomes more pronounced. We must recognize, however, that the degree and type of bacterial activity extends over, and may be responsible for, an infinitely wide range of varying symptoms.

The infection may be confined to comparatively narrow limits in the intestinal tract, or it may include the entire large intestine and the lower portion of the ileum. It may never invade the intestinal serosa, or it may produce extensive veils, bands and adhesions. The infection may be very mild and affect the epithelial cells of the mucous membrane only to a small degree, or it may affect the mucosa and the musculature and not the serosa. The character and activity of the germ life may be exceedingly mild or very severe. While some authors deny the existence of such a type of constipation as that recognized as spastic, it is true that certain types of irritation do exist wherein segments of the colon contract with unusual force and thus act as a source of mechanical obstruction to the progress of fecal material.

I have under observation at the present time a patient who has this condition in a most exaggerated degree. With the patient placed in the inverted position there is no difficulty in seeing one tightly contracting ring after another as the sigmoidoscope is introduced into the pelvic colon.

Again, the infection referred to often extends to the tissues of the anal outlet, and, as I have previously stated, it is at this point where we observe the most pronounced contractions to be found anywhere along the entire alimentary tract. In my opinion more stasis is produced in the large intestine and the terminal ileum by spastic contraction of these muscles than by any other factor entering into the etiology of constipation.

It is so seldom that examinations are made of the rectum, or rather the muscles controlling the rectal outlet, that it is quite impossible to estimate the magnitude of this phase of the subject. When due consideration is given this question it will be recognized that every abnormally contracted sphincter muscle is pathologic, and is a factor tending toward the production of constipation. The muscles may be very much hypertrophied and tightly contracted, and yet the 
patient may not complain of constipation. Under such circumstances, however, there are compensatory conditions existing higher in the intestinal canal whereby the contents are rendered either especially thin, the mucosa is unusually well lubricated, or some exaggerated lesion is present.

I will state that the chronic types of infection found at the rectal outlet are in some instances the most obstinate benign conditions that we have to contend with from the standpoint of effecting permanent relief, and this point should be borne in mind in all cases.

I have been especially interested in the source of these infections which play such an important part in constipation. It has been my observation that almost all of them have their origin in early infancy where the digestive apparatus remains deranged for a long period, or in some acute intestinal disease, such as typhoid fever, amebic infection, so-called flux, hookworm disease and other forms of infection. These attacks may have occurred years previously, yet it is significant when we note the large number of persons who have constipation and who give such histories.

Other enterocolonic lesions noted in constipation, with which you are familiar, and which have been extensively discussed during the last few years are veils, bands, adhesions and allied conditions. While these may be important factors in a certain number of instances, doubtless their significance has been greatly overestimated. In other words, there are many cases of obstinate constipation in which veils or bands are present, but if the other causative factors were removed and the veils allowed to remain, the patients would be completely relieved. Without making extensive reference to the cause of these bands and adhesions, it appears most logical that they are the results of bacterial activities, having their origin in the intestinal lumen during typhoid fever, amebic infection, flux and other inflammatory conditions. It seems to me that proof beyond peradventure has been established showing the relationship between these accessory tissues and the various infections just mentioned.

It is surprising to note the number of patients who complain of pain simulating appendicitis, in whom there is a previous history of acute intestinal infection followed, perhaps years later, by constipation, indigestion and other conditions resulting from bowel inactivity. At operation the blood vessels in the serous coat overlying the colon and appendix are found engorged, adhesions and veils are present, and the appendix.is removed without subsidence of the constipation and pain. These facts show the intimate relationship between the infection, constipation, bands, veils and other results of inflammation.

I wish to refer briefly to the question of ileocecal insufficiency. That there is more frequently a reflux of the cecal contents into the ileum than was formerly supposed cannot be doubted. In a very large number of patients it has been found that one out of six has ileocecal incompetency. An ingenious operation has been devised for the correction of this defect, but it has not been satisfactorily proved that the method always gives permanent relief. If stasis in the cecum and ascending colon is the chief factor in producing ileal regurgitation, then it is evident that the cause of the stasis should be determined and relieved.

I have referred to the powerful influence of the tightly contracted, irritable and hypertrophied muscles controlling the rectal outlet, the dry condition of the colonic mucosa and the other conditions recognized as factors in the production of constipation. If these conditions exert as potent an influence as suggested, and there can be no doubt that they are important forces in producing stasis in the right colon, then it would be logical treatment to remove the cause, after which it is more than probable the ileocecal defect would be corrected. I think this is a point at least worthy of consideration.

In conclusion, I wish to emphasize that we, as physicians, should view this subject in its broadest sense. We will be able in this way to glean the facts necessary for a comprehensive understanding of the question much more successfully than by studying it from a narrow and contracted standpoint.

\section{RECTAL CONDITIONS IN CHRONIC CONSTIPATION *}

ALOIS B. GRAHAM, A.M., M.D.

Captain, Medical Reserve Corps, U. S. Army

INDIANAPOLJS

Prima facie, nothing appears easier than the diagnosis of chronic constipation. Nevertheless, in not a few cases, it may be an exceedingly difficult task to determine accurately the etiologic factor. Many cases are due to mechanical causes which, not infrequently, are never recognized or even suspected. With the improved modern methods of examination at our command, physical, gastric analysis, examination of the stool, roentgenology, and proctologic examination, to which is added the clinical subjective signs and symptoms, an accurate diagnosis can and should be made in the majority of eases.

No case of chronic constipation is diagnosed correctly or should ever be treated as such, until a thorough proctologic examination has been made. The rectum, in many cases, is found distended with feces, the patient not experiencing the slightest sensation of its presence and having no desire whatever for stool. This rectal stasis may be atonic in character, or it may have its origin in definite rectal conditions. If atonic, it usually has its onset early in life. Repeated and prolonged inattention to the calls of nature, such as indolence, absolute disregard, and even resistance, obtund the nerves of the rectum so that they do not respond to stimulation. Murray attributes this loss of the sense of rectal pressure to "carelessness, ignorance and laziness."

Rectal conditions, as a rule, are easily diagnosed by a thorough proctologic examination. This examiniztion, by itself, is insufficient, and, in many cases, will not determine positively that the constipation has its origin in rectal conditions. I cannot emphasize too strongly the fact that identically the same rectal conditions may cause chronic constipation in one patient and yet have no appreciable influence in retarding the excretion of feces in another. If repeated digital examinations of a patient who has been given neither medicines nor enemas reveal a large amount of feces in the rectum, this alone should cause us to be suspicious of a rectal stasis. If the rectum is found to be almost or quite empty, we should feel reasonably certain that the constipation is due to delay higher up; and yet, we should not overlook the possibility of the

* Read before the Section on Gastro-Enterology and Proctology a the Sixty-Eighth Annual Session of the American Medical Association, New York, June, 1917. 